\title{
Total absorption studies of high priority decays for reactor applications: ${ }^{86} \mathrm{Br}$ and ${ }^{91} \mathrm{Rb}$
}

\author{
A. Algora ${ }^{1,2, a}$, S. Rice ${ }^{1,3}$, V. Guadilla ${ }^{1}$, J.L. Tain ${ }^{1}$, E. Valencia ${ }^{1}$, A.-A. Zakari-Issoufou ${ }^{4}$, J. Agramunt ${ }^{1}$, J. Äystö $^{5}$, \\ L. Batist ${ }^{6}$, J.A. Briz ${ }^{4}$, M. Bowry ${ }^{3}$, V.M. Bui ${ }^{4}$, R. Caballero-Folch ${ }^{7}$, D. Cano-Ott ${ }^{8}$, A. Cucoanes ${ }^{4}$, T. Eronen ${ }^{3}$, V.V. \\ V.S. Kolhinen ${ }^{5}$, F.G. Kondev ${ }^{12}$, J. Koponen ${ }^{5}$, M. Lebois ${ }^{13}$, T. Martinez ${ }^{8}$, P. Mason ${ }^{3}$, E. Mendoza ${ }^{8}$, M. Monserrate ${ }^{1}$, \\ A. Montaner-Pizá ${ }^{\text {, I. Moore }}{ }^{5}$, E. Nácher ${ }^{14}$, S.E.A. Orrigo ${ }^{1}$, H. Penttilä ${ }^{5}$, Z. Podolyák ${ }^{3}$, I. Pohjalainen ${ }^{5}$, A. Porta $^{4}$, \\ P.H. Regan ${ }^{3}$, J. Reinikainen ${ }^{5}$, M. Reponen ${ }^{5}$, S. Rinta-Antila ${ }^{5}$, J. Rissanen ${ }^{5}$, B. Rubio ${ }^{1}$, K. Rytkönen ${ }^{5}$, T. Shiba ${ }^{4}$, \\ V. Sonnenschein ${ }^{5}$, A.A. Sonzogni ${ }^{15}$, J.-Ch. Sublet ${ }^{10}$, V. Vedia ${ }^{9}$, A. Voss ${ }^{5}$, and J.N. Wilson ${ }^{13}$ \\ 1 Instituto de Física Corpuscular CSIC-Universidad de Valencia, 46071 Valencia, Spain \\ 2 Institute of Nuclear Research of the Hungarian Academy of Sciences, Debrecen 4026, Hungary \\ 3 Department of Physics, University of Surrey, GU2 7XH Guildford, UK \\ 4 Subatech, CNRS/INP2P3, Nantes, EMN, 44307 Nantes, France \\ 5 University of Jyvaskyla, Department of Physics, PO Box 35, 40014 University of Jyvaskyla, Finland \\ ${ }^{6}$ Petersburg Nuclear Physics Institute, 188300 Gatchina, Russia \\ 7 Universitat Politecnica de Catalunya, 08028 Barcelona, Spain \\ ${ }^{8}$ Centro de Investigaciones Energéticas Medioambientales y Tecnológicas, 28040 Madrid, Spain \\ 9 Universidad Complutense, Grupo de Física Nuclear, CEI Moncloa, 28040 Madrid, Spain \\ 10 United Kingdom Atomic Energy Authority, Culham Science Centre, Abingdon OX14 3DB, UK \\ 11 Department of Physics, Istanbul University, 34134 Istanbul, Turkey \\ 12 Nuclear Engineering Division, Argonne National Laboratory, Argonne, Illinois 60439, USA \\ 13 Institut de Physique Nucléaire d'Orsay, 91406 Orsay, France \\ 14 Instituto de Estructura de la Materia, CSIC, 28006 Madrid, Spain \\ 15 NNDC, Brookhaven National Laboratory, Upton, NY 11973-5000, USA
} Elomaa $^{3}$, E. Estevez ${ }^{1}$, M. Estienne ${ }^{4}$, M. Fallot ${ }^{4}$, G.F. Farrelly ${ }^{3}$, L.M. Fraile ${ }^{9}$, M. Fleming ${ }^{10}$, E. Ganioglu ${ }^{11}$, A.R. Garcia ${ }^{8}$, W. Gelletly ${ }^{1,3}$, B. Gómez-Hornillos ${ }^{7}$, D. Gorelov ${ }^{5}$, V. Gorlychev ${ }^{7}$, J. Hakala ${ }^{5}$, A. Jokinen ${ }^{5}$, D. Jordan ${ }^{1}$, A. Kankainen ${ }^{5}$,

\begin{abstract}
Preliminary results from beta decay studies of nuclei that are important for reactor applications are presented. The beta decays have been studied using the total absorption technique (TAS) and the pure beams provided by the JYFLTRAP system at the IGISOL facility of the University of Jyväskylä.
\end{abstract}

\section{Introduction}

Beta decay is an important source of nuclear structure information and can be used as a tool to study fundamental interactions. In addition, the study of beta decays, is also very relevant for practical and fundamental physics applications, such as the prediction of the decay heat from nuclear fuel and the prediction of the antineutrino spectrum from a working reactor $[1,2]$. For these two applications it is crucial to obtain experimental data that do not suffer from the Pandemonium effect [3], a systematic error associated with the use of conventional high-resolution spectroscopy techniques.

In this contribution we will present results from recently analyzed high priority beta decays [4] using the total absorption technique and discuss their impact on the prediction of the reactor decay heat. The measurements have been performed at the IGISOL facility of the University of Jyväkylä (Finland) using the high purity beams provided by the JYFLTRAP [5]. Where possible, comparisons will be given with earlier measurements

a e-mail: algora@ific.uv.es
[6] and with results obtained using different analysis techniques $[7,8]$, which will allow us to compare the correctness of the different methods, and validate their use.

The decay heat in reactors is usually defined as the amount of energy released by the decay of fission products without taking into account the antineutrinos. This can be estimated with summation calculations that imply determining the following power function $f(t)$ :

$$
f(t)=\sum_{i}\left(\bar{E}_{\beta, i}+\bar{E}_{\gamma, i}+\bar{E}_{\alpha, i}\right) \lambda_{i} N_{i}(t)
$$

where $\bar{E}_{i}$ is the mean decay energy of the $i^{\text {th }}$ nuclide $(\beta$, $\gamma$ and $\alpha$ components), $\lambda_{i}$ is the decay constant of the $i^{\text {th }}$ nuclide, and $N_{i}(t)$ is the number of nuclei $i$ at cooling time $t$. These calculations require extensive libraries of cross sections, fission yields and decay data.

The mean decay energies can be obtained by direct measurements as for example in Rudstam et al. [6] or can be deduced from decay data available in conventional databases like ENSDF [8]. For the latter, a knowledge of the feeding distribution to levels in the daughter nucleus is needed.

(C) The Authors, published by EDP Sciences. This is an Open Access article distributed under the terms of the Creative Commons Attribution License 4.0 (http://creativecommons.org/licenses/by/4.0/). 
As mentioned earlier, measurements of beta decays employing Ge detectors can suffer from the Pandemonium effect. In such conventional beta decay experiments the limited efficiency and sensitivity of Ge detectors can lead to incomplete decay schemes and to an incorrect determination of the beta feeding to levels in the daughter nucleus. Even using Ge arrays of very high efficiency this problem can still persist [9-11]. The solution to this problem is to use highly efficient devices or calorimeters, the so-called total absorption technique. Employing a large volume detector, usually constructed from scintillator materials of modest energy resolution, we can have total efficiencies of nearly $100 \%$ for detecting the gamma cascades that follow the beta decay. Extracting the information on the beta feeding from those experiments requires solving the following "inverse problem": $d=$ $R(B) . f$ where $d$ represents the measured spectrum free of contaminants, $R$ is the response matrix of the detector, and $f$ is the feeding distribution of the decay we wish to determine.

In this contribution we present preliminary results of the beta decay study of ${ }^{86} \mathrm{Br}$ and ${ }^{91} \mathrm{Rb}$ using the total absorption technique.

\section{Experiment and analysis}

The nuclei of interest were produced using proton induced fission in a ${ }^{238} \mathrm{U}$ target. The fission products were then extracted using the ion guide technique, which employs a helium jet to transport the reaction products to the first stage of the separator. After a first mass separation with the dipole magnet of moderate mass resolution of IGISOL $[12,13]$, the beams were isotopically separated further using the JYFL Penning trap [5].

The isotopically pure beams were then transported to the centre of the measuring setup. In the measurements presented here we used a segmented $\mathrm{BaF}_{2}$ total absorption spectrometer with a diameter and length of $25 \mathrm{~cm}$, and a longitudinal hole along its symmetry axis of $5 \mathrm{~cm}$ diameter. The radioactive beam was implanted in a tape system kept in vacuum, which was moved in cycles depending on the half-lives of the nuclei of interest. Behind the implantation point, at approximately $5 \mathrm{~mm}$ distance, a Si detector of $0.5 \mathrm{~mm}$ thickness was placed to record the beta particles in coincidence with the signals from the TAS spectrometer. The main goal of the experiment was to study the decay of nuclei that are important contributors to the decay heat and to the prediction of the antineutrino spectra in reactors. In this campaign special interest was devoted to decays that present beta-delayed neutron emission, in order to study the same decays with a combination of techniques that included the total absorption technique. Some of the results from this campaign have been already published or have been recently submitted for publication [14-17].

In the analysis of a total absorption measurement, the first concern is to determine the possible contaminants of the spectra to be analyzed (represented by $d$ in the inverse problem). In particular, the possible contributions of electronic pileup and daughter contamination have to be determined and quantified. The contamination of the daughter decay can be measured in a dedicated measurement or can be simulated if it arises from a well known decay. The pileup can be estimated using the procedure outlined in [18]. Then, the next step is to solve the above mentioned inverse problem. For that, the response function of the total absorption setup to the decay of interest has to be determined. This response (R) depends on the detector (geometry, materials, etc.) and on the branching ratios (B) of the levels populated in the decay. The branching ratio matrix is not known, and its calculation requires that one makes assumptions about the populated level scheme in the decay. Conventionally we accept levels and their gamma decay branches from high resolution measurements up to a certain cut-off energy. This is based on the assumption that low lying levels and their decay branches are relatively well known from high resolution measurements. Above the cut-off energy a statistical model is used to generate the branching ratio matrix between those levels (among themselves) and their connections to the low-lying part of the level scheme. Once the branching ratio matrix is defined, the response can be calculated recursively using previously validated Monte Carlo simulations. The analysis, which means to solve the inverse problem, is performed according to the methods developed by the Valencia group $[19,20]$.

\section{Study of the beta decay of ${ }^{86} \mathrm{Br}$ and ${ }^{91} \mathrm{Rb}$}

${ }^{86} \mathrm{Br}$ is considered priority one in the high priority list of beta decays of interest for decay heat calculations [4]. It decays to a stable nucleus, so in this particular case the only expected contamination in the measurements is the pileup.

For the determination of the response, we must first define the branching ratio matrix of the levels in the daughter nucleus. Levels and their decay branches up to the excitation of $3560 \mathrm{keV}$ were taken from the latest ENSDF compilation. Above that energy a statistical model based on level densities and gamma strength functions was used. More details are given in a forthcoming publication [17] and in [21].

In Fig. 1 we present the measured spectrum compared with the spectrum generated with the analysis (obtained by multipliying the corresponding response function with the resulting feeding distribution $R(B) . f^{\text {final }}$ ) and adding the contributions of the contaminants. In this analysis we had to include, apart from the pileup, an additional contaminant spectrum. A preliminary analysis of the pileup-cleaned spectrum showed that there is a small amount of contamination in the beta-gated spectra, which was due to an increased level of noise in the silicon detector in one of the runs. This was taken into account by subtracting from the beta-gated spectrum a background spectrum with beam-on, from which its own pileup had been previously subtracted. This small contribution is also presented in Fig. 1.

In Fig. 2 we present the accumulated feeding results from our analysis compared with the those taken from ENSDF [22]. This comparison shows clearly that previous measurements for this decay suffered from the Pandemonium effect.

From the preliminary feeding distribution obtained in the analysis we have calculated the mean energies, which are compared in Table 1 with the valued deduced from ENSDF (high resolution) and with the value from a recent publication by Fijałkowska et al. [23] who also used the TAS technique. 


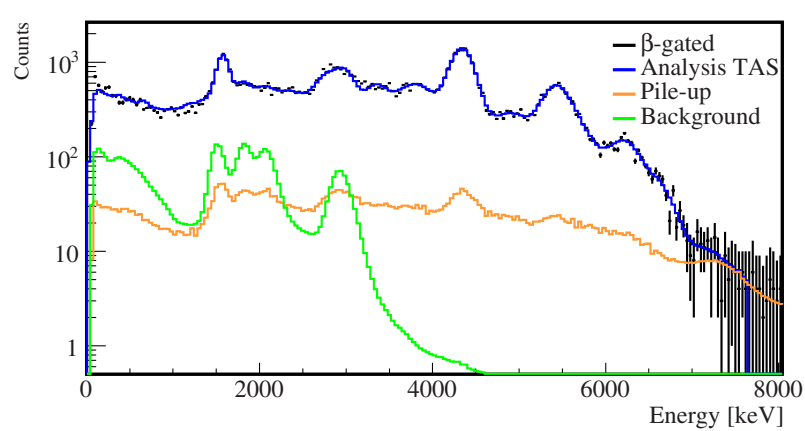

Figure 1. Comparison of the beta gated ${ }^{86} \mathrm{Br}$ decay TAS spectrum with the spectrum generated after the analysis. The contribution of the contaminants is also presented.

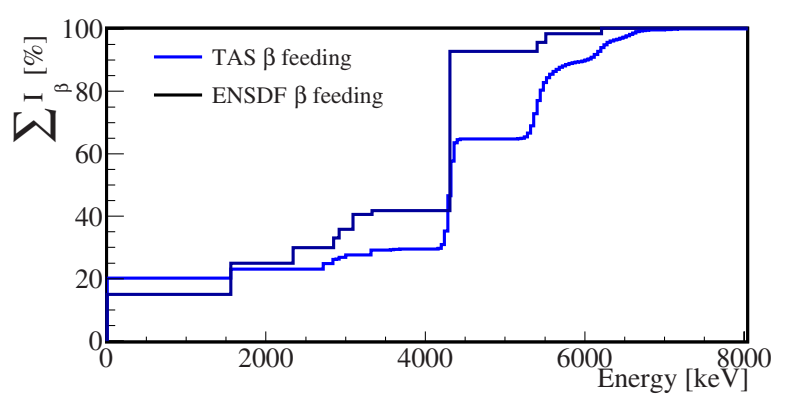

Figure 2. Accumulated feeding distribution from the analysis of the ${ }^{86} \mathrm{Br}$ decay compared with the feeding distribution available in ENSDF.

Table 1. Mean average energy for $\beta$-particles and $\gamma$ rays (all collected photons) from the decay of ${ }^{86} \mathrm{Br}$. The Oak Ridge result is taken from [23].

\begin{tabular}{lcc}
\hline & $\bar{E}_{\gamma}[\mathrm{keV}]$ & $\bar{E}_{\beta}[\mathrm{keV}]$ \\
\hline Present result & $3782(54)$ & $1687(28)$ \\
Oak Ridge result & $4110(<411)$ & \\
ENSDF & 3296 & 1944 \\
\hline
\end{tabular}

The decay of ${ }^{91} \mathrm{Rb}$ is not included in the high priority list [4], but it is interesting for other reasons. First of all it was also measured with the total absorption technique by Greenwood et al. [7], but analyzed using a different method. So, a comparison of the results obtained can allow us to draw conclusions on the results obtained by different methods of analysis and different setups. Secondly, this decay was used as a normalization point in the mean gamma energy measurements of Rudstam et al. [6], assuming that this decay was free from the Pandemonium effect. This is particularly relevant, since the Rudstam data set of mean gamma energies is one of the few available obtained by direct measurements and might require renormalization if the normalization point (decay of ${ }^{91} \mathrm{Rb}$ ) is proved to suffer from the Pandemonium effect.

In Fig. 3 we show a comparison of the measured TAS spectrum with the one generated from the feeding distribution obtained from the analysis [17,21]. In Fig. 4 we compare the accumulated feeding distribution with the measurements of Greenwood and with the high resolution results. Our results agree quite well with Greenwood results and show that this decay suffers from the Pandemonium effect. This implies that the data from Rudstam should be renormalized taking into account the newly determined mean energy.

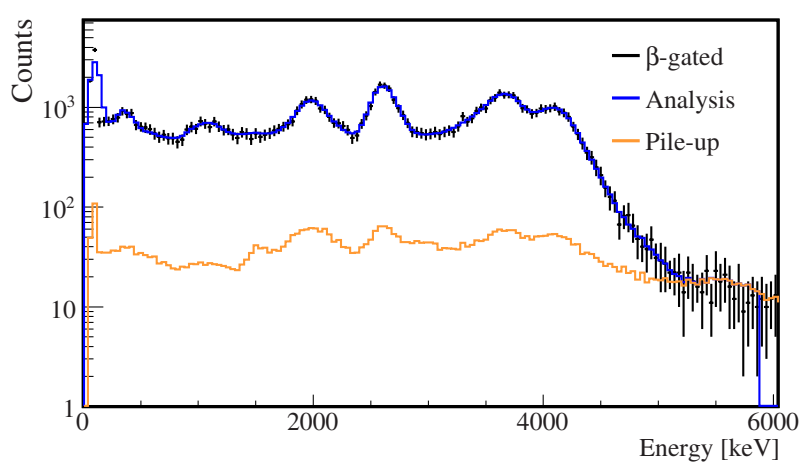

Figure 3. Comparison of the beta gated ${ }^{91} \mathrm{Rb}$ decay TAS spectrum with the spectrum generated after the analysis. The contribution of the contaminants is also presented in the figure.

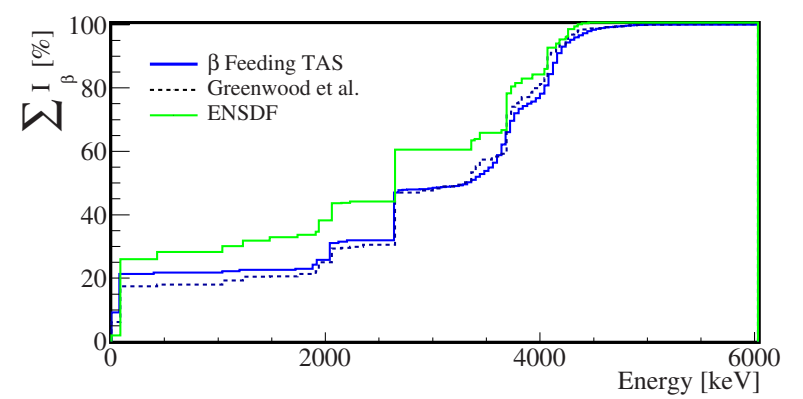

Figure 4. Accumulated feeding distribution from the analysis of the ${ }^{91} \mathrm{Rb}$ decay compared with the feeding distribution available in ENSDF and with the obtained by Greenwood et al. [7].

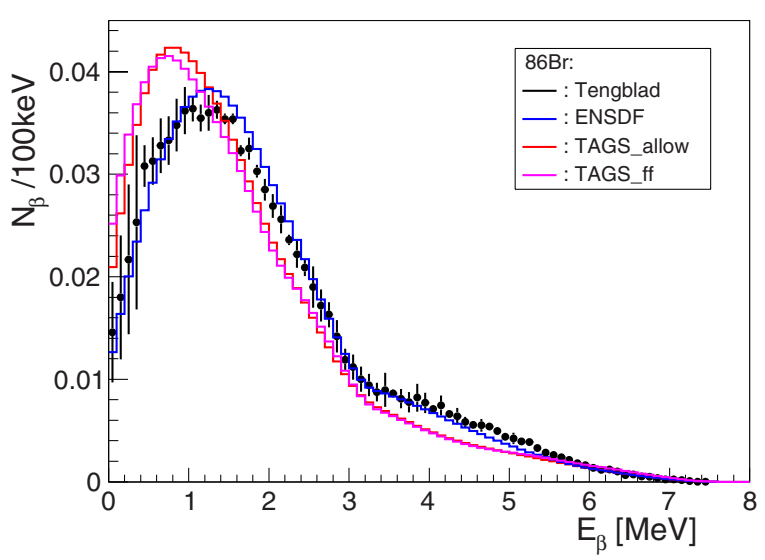

Figure 5. Comparison of the beta spectrum of the decay of ${ }^{86} \mathrm{Br}$ measured by Tengblad et al. [25] with the beta spectrum deduced from the TAS measurements. The effect of considering all beta transitions allowed or first forbidden in the deduction of the beta spectrum from the TAS measurements is also presented.

The feeding distributions can also be used to deduce the shape of the beta spectrum from this decay and to compare them with the direct measurements of Tengblad et al. [25]. In the cases presented here as well as in the cases studied in [15] we see a systematic difference. The beta spectrum deduced from the TAS measurements is softer (shifted to lower energies) than that measured by Tengblad et al. [25]. Several possible causes for these systematic differences have been explored (see [15]), but we do not see any possible reason arising from our analysis that could explain the large discrepancies. In Fig. 5 we show the comparison for the ${ }^{91} \mathrm{Rb}$ case. In the figure we also 


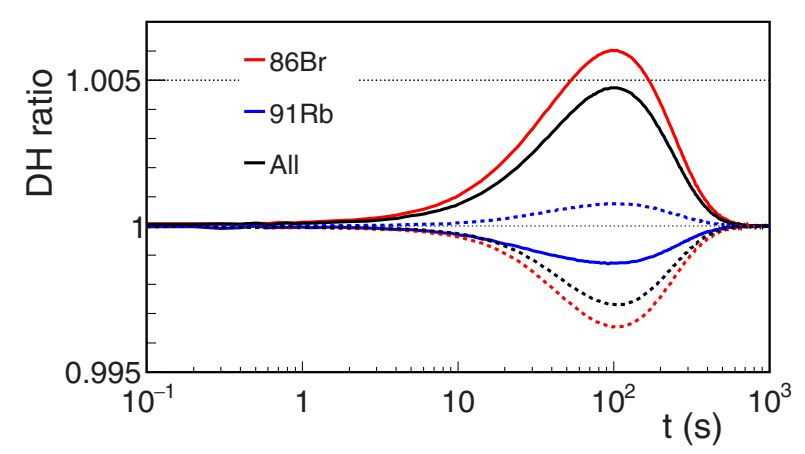

Figure 6. Relative contribution to the gamma and beta components of the decay heat of the presented decays $\left({ }^{86} \mathrm{Br}\right.$ and ${ }^{91} \mathrm{Rb}$ ) compared with the high-resolution contribution for ${ }^{235} \mathrm{U}$. The gamma component is represented by the continuous line. The beta component is showed with the dotted line.

present the effect of considering all beta transitions of first forbidden type in the calculated spectra deduced from the TAS measurements. Tengblad's results come from direct measurements, which means that they do not suffer from such assumptions. As can be seen from the comparison of the beta spectrum deduced from the TAS data using the allowed and the first forbidden assumption for all decays, it is clear that this supposition can not explain the differences with the Tengblad results. New direct measurements of beta spectra should be considered to further explore these differences.

The obtained mean energies from the TAS measurements can be used for decay heat calculations. The combined relative decay heat contributions of these results with respect to high resolution data is modest. It is of the order of $0.5 \%$ at its maximum for ${ }^{235} \mathrm{U}$ and $0.2 \%$ for ${ }^{239} \mathrm{Pu}$ for the electromagnetic component. The contribution to the gamma component relative to the high resolution values (using ENDF/B-VII.1) is presented in Fig. 6 for ${ }^{235} \mathrm{U}$. The relative contribution for the light particle (or beta component) is approximately $0.2 \%$ and $0.1 \%$ for ${ }^{235} \mathrm{U}$ and ${ }^{239} \mathrm{Pu}$ respectively at its maximum. The gamma and beta mean energies deduced from the present work for ${ }^{86} \mathrm{Br}$ are presented in Table 1, where they can be compared with the high resolution result and the recent measurement by the Oak Ridge group [23].

Summarizing, we have presented here two examples of decays studied by our TAS collaboration that were considered relevant for reactor applications [4]. ${ }^{91} \mathrm{Rb}$ is of particular interest, because it was used as a normalization point in the direct measurements of mean gamma energies performed by Rudstam et al.. Our study shows that both decays suffered from the Pandemonium effect.

This work was supported by Spanish Ministerio de Economía y Competitividad under grants FPA2008-06419, FPA2010-17142 and FPA2011-24553 and FPA2014-52823-C2-1-P, CPAN CSD2007-00042, and the program Severo Ochoa (SEV-2014-0398) and by EPSRC and STFC (UK). Work at ANL was supported by the U.S Department of Energy under contract DE-AC0206CH11357.

\section{References}

[1] A. Algora et al., Phys. Rev. Lett. 105, 202501 (2010)

[2] M. Fallot et al., Phys. Rev. Lett. 109, 202504 (2012)

[3] J. Hardy et al., Phys. Lett. B 71, 307 (1977)

[4] WPEC-25, Nuclear Science NEA, Report No. 6284, 2007; INDC(NDS)-0577(2010); INDC(NDS)0676(2015); INDC(NDS)-0499(2006)

[5] T. Eronen et al., Eur. Phys. J. A 48, 46 (2012)

[6] G. Rudstam et al., Atomic Data and Nuclear Data Tables 45, 239 (1990)

[7] R.C. Greenwood et al., Nucl. Instrum. Methods Phys. Res. A 390, 95 (1997)

[8] ENSDF, http://www.nndc.bnl.gov/ensdf

[9] A. Algora et al., Nucl. Phys. A 654, 727c (1999)

[10] A. Algora et al., Phys. Rev. C 68, 034301 (2003)

[11] Z. Hu et al., Phys. Rev. C 60, 024315 (1999)

[12] J. Äystö, Nucl. Phys. A 693, 477 (2001)

[13] I. Moore et al., Nucl. Instrum. Methods Phys. Res., Sect. B 317, 208 (2013)

[14] A.-A. Zakari-Issoufou et al., Phys. Rev. Lett. 115, 102503 (2015)

[15] E. Valencia et al., submitted to Phys. Rev. C.

[16] J.L. Tain et al., Phys. Rev. Lett. 115, 062502 (2015)

[17] S. Rice et al., to be submitted to Phys. Rev. C.

[18] D. Cano-Ott et al., Nucl. Instrum. Methods Phys. Res., Sect. A 430, 488 (1999)

[19] J.L. Tain et al., Nucl. Instrum. Methods Phys. Res. A 571, 719 (2007)

[20] J.L. Tain et al., Nucl. Instrum. Methods Phys. Res. A 571, 728 (2007)

[21] S. Rice, PhD thesis, University of Surrey, 2014

[22] A. Negret, B. Singh, Nuclear Data Sheets 124, 1 (2015)

[23] A. Fijalkowska et al., Acta Phys. Polonica B 45, 545 (2014)

[24] C.M. Baglin, Nuclear Data Sheets 114, 1293 (2013)

[25] O. Tengblad et al., Nucl. Phys. A 503, 136 (1989) 